ENCYCLOPEDDIE Encyclopédie berbère

BERBERE

32 | 2010

32 | Mgild - Mzab

\title{
Monnaie
}

\section{J. Alexandropoulos}

\section{OpenEdition}

Journals

Édition électronique

URL : https://journals.openedition.org/encyclopedieberbere/625

DOI : 10.4000/encyclopedieberbere.625

ISSN : 2262-7197

\section{Éditeur}

Peeters Publishers

\section{Édition imprimée}

Date de publication : 31 décembre 2010

Pagination : 5068-5076

ISBN : 978-90-429-2369-0

ISSN : 1015-7344

\section{Référence électronique}

J. Alexandropoulos, « Monnaie », Encyclopédie berbère [En ligne], 32 | 2010, document M128, mis en ligne le 11 novembre 2020, consulté le 17 février 2022. URL : http://journals.openedition.org/ encyclopedieberbere/625; DOI : https://doi.org/10.4000/encyclopedieberbere.625

Ce document a été généré automatiquement le 17 février 2022.

(c) Tous droits réservés 


\section{Monnaie}

\section{J. Alexandropoulos}

1 Les royaumes numides* et maurétaniens* ont frappé monnaie sur une période d'environ 250 ans, comprise entre la Deuxième Guerre Punique (218-201 av. J.C.) et l'achèvement de la conquête romaine du Maghreb (annexion des Maurétanies* en 40 ap. J.C.). Cette période correspond à la structuration des anciennes confédérations de tribus berbères en Etats de type hellénistique jusqu'à leur passage progressif sous l'obédience romaine. Le phénomène monétaire accompagne ces évolutions, mettant en évidence l'affirmation du pouvoir royal, le développement de l'économie monétaire africaine et le rayonnement des diverses influences - punique, hellénistique, romainequi ont contribué à modeler la personnalité de ces royaumes au moment de leur pleine intégration à la Méditerranée de leur temps. On distingue parmi ces monnayages les frappes royales et les émissions des diverses cités.

\section{Les monnayages royaux}

2 Des contextes monétaires divers ont marqué la naissance et le développement des monnayages royaux. Ils apparaissent, au moment de la Deuxième Guerre Punique, et reçoivent pour l'essentiel une influence monétaire punique double. L'une vient de l'Est, de Carthage même, dont les monnaies se caractérisent par l'emploi, pour les trois métaux, du shekel de 7,60 g comme étalon monétaire. Leur iconographie comporte à l'avers l'effigie d'une déesse couronnée d'épis où l'on peut reconnaître une forme hellénisée de la déesse Tanit*, et au revers un cheval aux allures diverses suivant les émissions, voire parfois un simple protomé de cheval. Lorsqu'apparaissent les premières monnaies numides, ces émissions de Carthage circulent largement dans son hinterland africain, mais aussi dans les cités de la côte orientale du royaume numide. La deuxième influence monétaire, punique également, vient d'Espagne où circulent les émissions d'anciennes cités phénico-puniques comme Gadès et celles des généraux barcides qui frappent monnaie sur place pour les besoins de leurs troupes. Toutes ces émissions hispaniques se caractérisent par des étalons particuliers : étalon "ibérique » de $4,80 \mathrm{~g}$ ou shekel hispanique de 7,20g pour l'argent, et unité de $8 \mathrm{~g}$ puis de $10-11 \mathrm{~g}$ 
pour le bronze. L'iconographie est variée. Sur les émissions des cités domine le thème de Melqart gaditain sous les traits d'Héraklès, accompagné de symboles solaires, agricoles (épi de blé) ou maritimes (thons). Les émissions barcides reprennent parfois l'effigie carthaginoise de Tanit et le thème du cheval, mais y rajoutent des thèmes plus militaires, comme Héraklès-Melqart dans sa fonction combattante, la galère, l'éléphant ou le casque. Toutes ces monnaies circulent en abondance en Espagne et certaines, vraisemblablement, dans les villes côtières phénico-puniques de Maurétanie.

\section{La Numidie}

3 L'émission des premières monnaies numides accompagne l'entrée sur la scène méditerranéenne de Syphax* au moment de la Deuxième Guerre Punique. Sollicité par les deux camps et ayant échoué dans son rôle d'arbitre il se rallie à Carthage et prend une part active à la guerre, éliminant provisoirement son rival massyle*, Massinissa*. Dans ce contexte deux séries de bronze sont émises, sans doute à Siga, portant au droit l'effigie nue ou diadémée de Syphax et au revers un cavalier au galop portant les insignes royaux avec la légende punique SPQ HMMLKT (figure 1) L'étalon utilisé vient d'Espagne. Ces émissions peu volumineuses entament modestement la monétarisation du royaume. Il y eut *peut-être des frappes d'argent qui ne nous sont pas parvenues. Pour Verminad*, fils de Syphax (figure 2), il existe des émissions de bronze très proches des précédentes et de même métrologie : Tête diadémée du roi au droit, et cheval au galop au revers avec la légende punique WRMND HMMLKT. Pour ces monnaies comme pour les rares émissions d'argent semblables, frappées selon la métrologie du shekel, l'incertitude demeure sur la date d'émission: du vivant de Syphax ou dans un royaume laissé par Rome à Verminad après la capture de Syphax ?

Mais la véritable monétarisation du royaume commence avec Massinissa*. Il existe une masse considérable de bronzes numides d'un diamètre d'environ $27 \mathrm{~mm}$ pour un poids de 14-15 g, portant au droit l'effigie laurée de ce roi et au revers un cheval au galop (figure 3). Ces monnaies, qui constituent l'unité du système métrologique numide oriental, se retrouvent d'un bout à l'autre du Maghreb et forment, avec les monnaies puniques de Carthage pour l'Est de la région, l'essentiel de la masse monétaire en circulation en Afrique jusqu'à l'époque augustéenne. Les plus anciennes de ces unités numides portent des lettres puniques indiquant le nom de Massinissa (203-148 av. J.C.) ou Micipsa* (148-118 av. J.C.) d'autres, celui de Gulussa (148- ? av. J.C.) ou Gauda (105- ? av. J.C.), et d'Adherbal (118-112 av. J.C.). Mais la grande majorité est anépigraphe et leur nombre laisse entrevoir des émissions très abondantes sur une très longue durée, sans doute jusqu'au règne de Juba 1er $(60$ ?-46 av. J.C.) qui réformera fortement le monnayage numide. Ces unités sont très proches de modèles carthaginois utilisés durant et après la deuxième guerre punique (figure 4) tant par la métrologie que par l'iconographie ou la technique de frappe et prévues manifestement pour une circulation commune, au départ dans la partie orientale du Maghreb. L'atelier principal est donc très vraisemblablement Cirta, mais l'ampleur des frappes rend probable l'existence d'ateliers secondaires encore inconnus. Il existe de rares multiples de ces unités numides, sans doute datables du règne de Massinissa, de même que des sousmultiples un peu plus fréquents. Toutes ces espèces divisionnaires présentent au droit l'effigie de Massinissa et au revers un cheval aux allures diverses selon la valeur. On mettra à part une émission sans doute inaugurale de Massinissa montrant son effigie avec ou sans les insignes royaux, et au revers le nom du roi en toutes lettres, MSNSN 
HMMLKT, au-dessous d'un cheval ou d'un éléphant (figures 5 et 6). La diffusion des unités de $27 \mathrm{~mm}$ frappées à Cirta est donc générale au Maghreb, même si un temps sans doute sous Massinissa et peut-être une partie du règne de Micipsa - l'atelier de Siga a continué à frapper pour ces souverains des monnaies selon l'ancienne métrologie punico-hispanique de Syphax avec l'effigie diadémée du souverain (figure 7).

On remarquera que seules des monnaies de bronze semblent avoir été frappées sous Massinissa et ses successeurs jusqu'à Juba Ier. Nous n'en avons pas d'or ni d'argent. La circulation des monnaies d'argent était surtout assurée par les deniers romains qu'apportait le commerce des negotiatores italiens en Numidie. Le trésor de Cirta, enfoui après $79 \mathrm{av}$. J.C. montre bien cette prédominance du denier à côté d'autres espèces plus diverses : 136 deniers romains pour 75 deniers celtibères, 14 tétradrachmes athéniens et 12 drachmes massaliotes. Il existait aussi de nombreuses monnaies (?) de plomb du type de l'unité de $27 \mathrm{~mm}$, dont il est difficile de savoir le statut exact: monnaie frauduleuse d'imitation, monnaie officielle de temps de pénurie ou encore monnaie votive?

Notons qu'il n'existe aucune monnaie attribuable à Jugurtha* (118-105 av. J.C.) et que celles que l'on attribue à Capussa* (cousin de Massinissa et son prédécesseur sur le trône de Massylie) sont certainement d'époque postérieure.

7 Avec Juba I ${ }^{\text {er* }}$ (60 ?-46 av. J.C.) des modifications décisives interviennent dans le monnayage numide. D'abord avec l'introduction d'un vrai bimétallisme. A Cirta même, de petites divisions d'argent (quinaire et sesterce d'étalon romain) sont frappées à partir de ressources métalliques manifestement réduites. Mais à Utique, durant la campagne de 47-46 et sans doute par des prélèvements sur le trésor de guerre de ses alliés pompéiens, Juba fait frapper de nombreux deniers à son effigie (figure 8). D’autre part, l'atelier de Cirta émet des monnaies qui forment dans leur ensemble une échelle divisionnaire complète, de même métrologie que les bronzes numides antérieurs et comprenant une unité de $27 \mathrm{~mm}$, un demi de $22 \mathrm{~mm}$ et un multiple de $35 \mathrm{~mm}$ (figures 9-11). Ces monnaies sont de billon ou de bronze.

8 L'iconographie de toutes ces émissions renouvelle complètement la tradition monétaire numide : effigie de Zeus Ammon, de l'Afrique coiffée de la trompe d'éléphant, lion passant, éléphant, monuments tels que temple ou palais. L'effigie du roi avec les attributs royaux est soignée et sa titulature, parfois bilingue punique et latine, insiste sur le titre de REX/HMMLKT. Toutes ces nouveautés témoignent d'emprunts à l'Egypte lagide et de la reconnaissance politique que Juba a su obtenir de la Rome pompéienne en échange de son alliance. Ce monnayage reflète toutes les évolutions du royaume à la veille son annexion par Rome (46 av. J.C.).

9 Notons pour finir qu'il existe des monnayages royaux numides et/ou maurétaniens du ${ }^{\mathrm{I} r}$ s. av. J.C. au nom d'un ou deux MŠTNȘN (?) d'attribution totalement incertaine.

\section{La Maurétanie}

La Maurétanie se monétarise plus tard que la Numidie. C'est sans doute à Bocchus I ${ }^{\text {er* }}$ (118 ?-80 ? av. J. C.) que l'on doit les premières émissions royales maurétaniennes. Elles portent le nom BQŠ en écriture néo-punique et un portrait rudimentaire de Bocchus $\mathrm{I}^{\mathrm{er}}$ (figure 12). Au revers de ces monnaies frappées à Siga et ŠMŠ selon l'étalon traditionnel de la région (unité de 10-11g déjà utilisée par Syphax, puis Massinissa à Siga) sont figurés une grappe de raisin ou le dieu Bacchus, par jeu de mots avec Bocchus ou en 
raison de la célébrité des vignes locales. Par la suite, les monnaies frappées à Rusaddir, Tamuda, Tingi, Sala, ŠMŠ, Camarata (?) et Timici portent invariablement l'effigie royale sans nom de souverain et des symboles de fertilité (épi et grappe de raisin) ou astraux. On ne peut donc les attribuer avec certitude à tel ou tel règne.

On ne saurait utiliser la numismatique pour démêler ensuite la succession des rois de Maurétanie, puisque seuls Bogud* en Maurétanie occidentale (49-38 av. J.C.) et Bocchus II (49-33 av. J.C.) en Maurétanie orientale sont attestés comme souverains émetteurs pour l'époque des guerres civiles romaines. Bogud frappe de beaux mais rares deniers au type de l'Afrique ou à thèmes solaires : griffon et disque ailé (figure 13). Ses monnaies de bronze montrent son portrait et un revers identique aux as républicains de Rome: une galère. On ne connaît que des monnaies de bronze pour Bocchus II, portant l'effigie royale et des motifs animaliers africains : éléphant, lion et cheval. Une émission imite elle aussi les as républicains puisque l'effigie royale y est janiforme (figure 14). Elle est accompagnée d'une représentation de l'Afrique. Pour toutes ces monnaies de Bogud et Bocchus II l'atelier émetteur est incertain.

Mais le grand développement de la monnaie maurétanienne s'accomplit sous Juba II* (25 av. J.C.-23 ap. J.C.) et son fils Ptolémée* (23 - 40 ap. J.C.). A l'ombre de la domination monétaire du denier romain, et après une période "d'interrègne " (33-25 av. J.C.) durant laquelle on frappe à Iol de rares bronzes à l'effigie d'Octave accompagnée de thèmes africains (tête de l'Afrique, lion et éléphant), Juba II lance de nombreuses émissions qui accomplissent la synthèse entre les diverses influences monétaires qui se sont exprimées jusque là en Afrique, tout en rapprochant son monnayage de celui de Rome. Le trimétallisme apparaît pour la première fois dans les royaumes numidomaurétaniens. De très rares frappes d'or à l'effigie de Juba II et célébrant son épouse Cléopâtre, sont produites selon un étalon romain réduit (figure 15). Leur tout petit nombre en fait sans doute surtout des émissions de prestige. En revanche, un volume considérable de monnaies d'argent est émis, là encore selon un étalon romain qui se réduit au fil du règne. Il s'agit donc de "deniers » dont le cours était inférieur à leurs homologues de Rome. Ces deniers circulent largement de l'Atlantique jusqu'à l'Est de Caesarea qui est sans doute l'atelier de frappe. On en retrouve jusqu'en Numidie (trésor du Cap Djinet). L'iconographie de ces deniers montre une effigie de Juba II diadémée ou couverte de la léonté et parfois accompagnée d'une massue (figure 16). Les revers, d'iconographie très variée renvoient à des thèmes africains (tête d'Africa, lion, éléphant), égyptiens (symboles isiaques, crocodile, vache, corne d'abondance), ou rappelant la loyauté du roi à l'égard de Rome (temple en l'honneur d'Auguste, Capricorne). D'autres montrent les insignes royaux ou célèbrent les victoires africaines de Juba. Les langues utilisées sont le latin et le grec, le punique a disparu. Avec le monnayage de bronze de Juba II on retrouve les mêmes éléments de synthèse. L'iconographie, variée, reprend la plupart des thèmes existants pour l'argent, et le bilinguisme gréco-latin des légendes est identique. La métrologie est plus complexe. L'unité de base du système reprend les caractéristiques des bronzes de Massinissa antérieurs qui constituaient l'essentiel de la masse monétaire en circulation. Néanmoins de très nombreux sous-multiples (demis et quarts d'unité) sont créés, compatibles avec les petites divisions émises auparavant sous Bocchus $I^{\text {er }}$ et ses successeurs. Et surtout on voit apparaître des doubles et quadruples d'unité dont le modèle métrologique est à chercher auprès des monnayages romanisés de Gadès. C'est donc une échelle divisionnaire très complète, abondante, compatible avec les espèces 
déjà en circulation et d'iconographie variée qui est mise en circulation. L'image du pouvoir est ainsi largement diffusée, sans doute essentiellement à partir de l'atelier de Caesarea, dans la partie monétarisée du royaume. Dans le monnayage comme ailleurs, s'exprime la synthèse culturelle méditerranéenne qui caractérise la monarchie de Juba II et Cléopâtre.

13 Avec Ptolémée* (23-40 ap. J.C.), les caractéristiques essentielles du monnayage de Juba II demeurent, donnant cependant l'impression d'une moindre vitalité. Les monnaies d'or restent exceptionnelles, célébrant le pouvoir d'Auguste et celui de Ptolémée (insignes royaux). Le monnayage d'argent décline en quantité et en qualité (figure 17). Les « deniers » sont plus légers et l'iconographie s'appauvrit. On y retrouve les thèmes africains (tête d'Africa, cheval, lion, éléphant), africano-égyptiens ou égyptiens (palmier, corne d'abondance), romains (temple) ou auto-célébrants (insignes royaux). Le monnayage de bronze est plus intéressant et montre deux périodes. Une première qui reprend les modalités de celui de Juba II de manière affaiblie : seuls l'unité et ses sous-multiples sont émis, en quantités inférieures et avec une iconographie restreinte pour l'essentiel au portrait royal, accompagné du lion et de symboles astraux. Mais dans les deux dernières années du règne on voit apparaitre un véritable portrait royal, loin de l'idéalisation progressivement abâtardie que l'on connaissait depuis les débuts de Juba II. La titulature est plus riche, indiquant la filiation depuis Juba Ier. Et surtout la métrologie se modifie, se rapprochant de celle de Rome (figure 18). Le monnayage de Ptolémée semble avoir connu la même diffusion que celui de Juba II.

\section{Les monnayages poliades}

14 Sur le territoire des royaumes numides et maurétaniens, le monnayage royal n'est pas en situation de complet monopole. Certaines cités ont le leur, métrologiquement articulé avec les monnayages dominants. Le plus ancien numéraire poliade, celui d'Iol, apparu durant la Deuxième Guerre Punique, portait à l'avers l'effigie d'Isis et au revers trois épis de blé (figure 19). Il s'agit essentiellement de monnaies de bronze émises en grandes quantités à côté de très rares espèces d'argent qui suivent la métrologie du shekel. D'autres villes de Numidie ont émis des monnaies, de bronze uniquement, d'une diffusion essentiellement locale. Hippo Regius (si c'est bien de cette ville qu'il s'agit et non d'Hippo Diarrhytus) frappe d'abord dans la $2 \mathrm{e}$ moitié du $\mathrm{I}^{\mathrm{er}}$ s. av. J.C. des monnaies marquées 'PWN en néo-punique à l'effigie des dieux poliades: un Baal barbu et lauré, Isis, Hercule, Vulcain et Bacchus. Viennent ensuite à l'époque augustéenne et tibérienne des frappes marquées HIPPONE LIBERA en latin, aux effigies impériales accompagnées de Junon Caelestis et Cérès. Le style en est très proche des frappes des cités d'Afrique proconsulaire.

Cirta émet également deux groupes de monnaies. Un premier, datable sans doute des derniers temps de Juba $\mathrm{I}^{\mathrm{er}}$ ou des premiers de l'annexion, marqué en néo-punique KRṬN avec une effigie de Tyché, une porte de cité ou un cheval, le tout accompagné du nom des suffètes (figure 20). D'autres monnaies suivent immédiatement : elles montrent le portrait du condottiere P. Sittius et au revers des allégories ou divinités: Honos et Virtus, Minerve, Jupiter ( ?) (figure 21). Le premier ensemble, le plus abondant, eut une diffusion locale. Le second apparaît comme une émission de circonstance. La cité de Macomades eut peut-être un faible monnayage aux types de la Tyché et du cheval. Les émissions d'Icosium (fin $\mathrm{II}^{\mathrm{e}}$ s. av. J.C.) nous sont essentiellement connues par un trésor 
trouvé sur place, contenant des monnaies de bronze et de plomb, marquées 'WYKSM et toutes aux même types: une tête d'Isis couronnée par une victoire et un baal coiffé d'une tiare à plumes (figure 22). La ville de Gunugu émet au I ${ }^{\mathrm{er}}$ s. av. J.C. de rares monnaies portant l'ethnique GNGN en écriture néopunique, l'effigie de Bacchus (?) et d'un dieu à tiare, semblable à celui d'Icosium, flanqué d'un caducée.

L'atelier de Caesarea, outre son rôle d'émetteur sans doute unique des monnayages royaux de Juba II et Ptolémée, produisit quelques frappes célébrant la ville elle-même sous les traits de Tyché accompagnée de la légende SITVM CAESARIS, rappelant l'origine de son nouveau nom (figure 23).

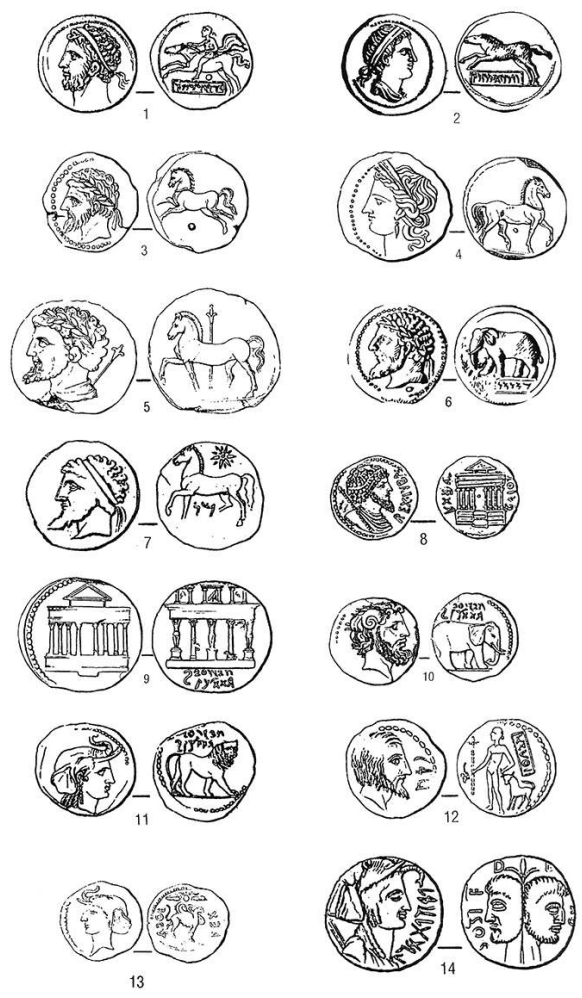

PI. MONNAIES NUMIDES ET MAURÉtANIENNES. 


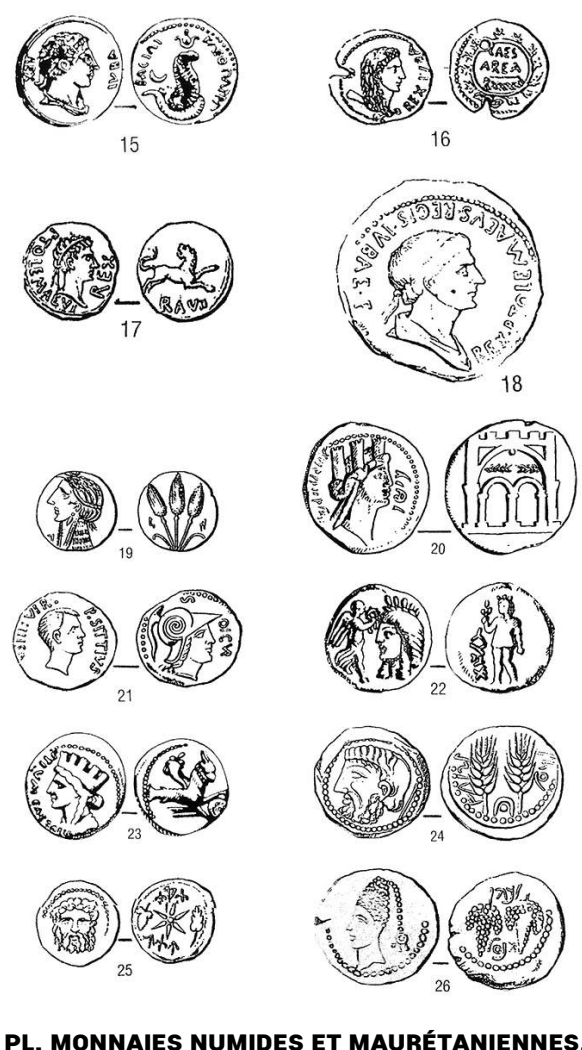

Dans la moitié occidentale du Maghreb, les cités ont souvent frappé à l'époque préromaine ( $\mathrm{e}^{\mathrm{er}} \mathrm{s}$. av. J.C.), des monnaies portant des symboles locaux (blé, vigne, abeille) ou astraux, associés à l'image royale. Si l'on excepte Cartenna dont on ne connaît qu'une émission d'époque impériale romaine, c'est le cas pour Timici, Camarata (?), Rusaddir, Tamuda ou encore Sala. On ne saurait évaluer la durée de ces frappes. Des monnaies du $\mathrm{I}^{\mathrm{er}}$ s. av. J.C., portant un aigle, des symboles astraux et la légende punique BBCL pourraient appartenir à Volubilis*. Toutes ces émissions ont eu une circulation très limitée.

18 Les ateliers de Tingi*, Lixus et ŠMŠ assurent, en fait, la part principale des frappes poliades de Maurétanie occidentale. Leur métrologie est calquée sur celle des monnaies de Gadès, autour d'une unité de 12-13g (atelier de Tingi surtout : figure 24) accompagnée de nombreux demis et quarts (pour les trois ateliers) et de rares multiples (Tingi uniquement). Les légendes sont parfois bilingues, puniques et latines. La localisation de l'atelier de ŠMŠ (figure 25) reste en question. Il est traditionnellement considéré comme identifiable à Lixus, mais les provenances pourraient suggérer une identification à Volubilis. Toutes ces frappes couvriraient la deuxième moitié du $\mathrm{I}^{\mathrm{er}} \mathrm{s}$. av. J.C. et peut-être l'ensemble du règne de Juba II. L'iconographie dans son économie générale, de même que les légendes, empruntent beaucoup au monnayage gaditain, surtout à Tingi où les représentations d'Océan rappellent celles de Melqart au nord du Détroit. Le dieu à bonnet conique de Lixus (figure 26) reste plus mystérieux. Des symboles astraux ou liés aux ressources locales (blé, vigne, thons) se retrouvent sur les trois monnayages. L'image impériale augustéenne reste cantonnée à Tingi. Les ateliers de Iulia Campestris (Babba) et Zilil, en revanche consacrent l'essentiel de leurs rares émissions à la célébration d'Auguste et de leur statut municipal. Toutes ces frappes poliades maurétaniennes cesseront dès avant l'annexion romaine. 
19 Les monnaies numides et maurétaniennes, tant royales que poliades, continueront à être utilisées dans la circulation monétaire africaine jusque bien avant dans la période romaine, mêlées à celles de l'atelier de Rome, car ce n'est qu'à l'époque de Domitien et surtout d'Hadrien que les frappes de Rome alimenteront l'Afrique des quantités de numéraire nécessaires aux échanges locaux.

\section{BIBLIOGRAPHIE}

ALEXANDROPOULOS J., Les monnaies de l'Afrique antique, Toulouse, 2000.

AMANDRY M., BURNeTT A., RIPOLES P. P., Roman Provincial Coinage, I. From the Death of Caesar to the Death of Vitellius (44 B.C.-69 A.C.), Paris-Londres, 1992.

MAZARD J., Corpus, Nummorum Numidiae Mauretaniaeque, Paris, 1955.

MÜLLER L., Numismatique de l'ancienne Afrique, Copenhague, 1860-1874.

INDEX

Mots-clés : Antiquité, Commerce, Economie, Histoire, Maurétanie, Numidie, Punique 